\title{
Difficulties in applying pure Kohn-Sham density functional theory electronic structure methods to protein molecules
}

\author{
Elias Rudberg* \\ Division of Scientific Computing, Department of Information Technology, \\ Uppsala University, Box 337, SE-751 05 Uppsala, Sweden
}

(Dated: May 23, 2018)

\begin{abstract}
Self-consistency based Kohn-Sham density functional theory (KS-DFT) electronic structure calculations with Gaussian basis sets are reported for a set of 17 protein-like molecules with geometries obtained from the protein data bank. It is found that in many cases such calculations do not converge due to vanishing HOMO-LUMO gaps. A sequence of polyproline I helix molecules is also studied, and it is found that self-consistency calculations using pure functionals fail to converge for helices longer than six proline units. Since the computed gap is strongly correlated to the fraction of Hartree-Fock exchange, test calculations using both pure and hybrid density functionals are reported. The tested methods include the pure functionals BLYP, PBE, and LDA, as well as Hartree-Fock and the hybrid functionals BHandHLYP, B3LYP, and PBE0. The effect of including solvent molecules in the calculations is studied, and it is found that the inclusion of explicit solvent molecules around the protein fragment in many cases gives a larger gap, but that convergence problems due to vanishing gaps still occur in calculations with pure functionals. In order to achieve converged results, some modeling of the charge distribution of solvent water molecules outside the electronic structure calculation is needed. Representing solvent water molecules by a simple point charge distribution is found to give non-vanishing HOMO-LUMO gaps for the tested protein-like systems also for pure functionals.
\end{abstract}

\section{INTRODUCTION}

Kohn-Sham density functional theory [1] (KS-DFT) has been widely used in electronic structure calculations. Efficient algorithms have been developed that allow KS-DFT methods to be

\footnotetext{
*Electronic address: elias.rudberg@it.uu.se
} 
applied for large molecules, including hundreds and even thousands of atoms [2]. However, a problem with KS-DFT is the self-interaction error (SIE). For example, it is known that SIE causes severe errors in computed polymer polarizabilities [3] where the problem becomes more and more severe with increasing system size. Thus, application of KS-DFT to large systems is not always straightforward.

One important application of KS-DFT for large molecules is the study of proteins, whose properties are of interest in biology. In this work, we study the applicability of standard self-consistency based KS-DFT methods for calculations on protein molecules.

\section{METHOD}

In KS-DFT methods, the electron density is expressed via a set of orbitals in a similar way as in the Hartree-Fock [4] (HF) method. We consider here non-periodic spin-restricted KS-DFT methods at zero electronic temperature. Then, the number of occupied orbitals is $n_{\text {occ }}=n / 2$ where $n$ is the number of electrons in the system. The Kohn-Sham orbitals are determined by solving

$$
\mathbf{F C}=\mathbf{S C} \epsilon
$$

where $\mathbf{F}$ is the Kohn-Sham matrix, $\mathbf{C}$ the matrix of orbital coefficients, $\mathbf{S}$ the overlap matrix and $\epsilon$ the diagonal matrix of orbital energies. The matrices in (1) are $N \times N$ matrices, where $N$ is the number of basis functions. Given a set of $N$ orbitals that constitute a solution to (1), a set of occupied orbitals is formed by including the $n_{\text {occ }}$ orbitals of lowest energy. The occupied orbitals determine the density matrix $\mathbf{D}$ as

$$
D_{i j}=2 \sum_{k=1}^{n_{\text {occ }}} C_{i k} C_{j k}
$$

where the columns of $\mathbf{C}$ are taken to be ordered by the corresponding orbital energies. The KohnSham matrix $\mathbf{F}$ is computed from $\mathbf{D}$ according to the chosen exchange-correlation functional. Since $\mathbf{F}$ depends on $\mathbf{D}$, an iterative procedure is used to find a self-consistent solution.

Calculations where a new density matrix is computed by occupying the orbitals of lowest energy as described above are in this work referred to as self-consistency based calculations, to clearly distinguish them from direct minimization approaches.

In self-consistency based calculations, convergence schemes such as damping [5] and DIIS [6] are usually employed, where a new Kohn-Sham matrix is constructed by taking information from 
previous iterations into account. See the work of Kudin and Scuseria [7] for an overview of such convergence schemes.

The self-consistency based approach usually works well provided that there is a sizable gap between the highest occupied molecular orbital (HOMO) and lowest unoccupied molecular orbital (LUMO) energies. However, there is no guarantee that the HOMO-LUMO gap will be large; the gap depends on the studied system as well as on the basis set and on the used exchange-correlation functional. If the gap is very small the procedure of determining the occupied orbitals needed in (2) becomes ill-defined and then a self-consistent solution may be difficult or even impossible to find.

KS-DFT exchange-correlation functionals can be divided into two main classes: pure and hybrid functionals. In hybrid functionals, some fraction of HF exchange is added to the Kohn-Sham matrix, often using empirically determined constants.

\section{RESULTS}

This section includes results of HF and KS-DFT calculations for various protein-like systems. The presented results were computed using the Ergo program [8]. The obtained results can however be readily reproduced using other KS-DFT codes employing Gaussian basis functions.

The convergence scheme used in the reported calculations is a combination of damping and DIIS, as implemented in the Ergo program. This scheme essentially uses damping in early iterations, with a dynamically adapted damping factor such that the step size is decreased whenever the energy goes up, leading to very small steps in difficult cases. However, details of this scheme do not affect the reported results; the observed convergence problems are due to vanishing HOMOLUMO gaps, a problem that neither damping nor DIIS-like schemes can resolve.

In this work, calculations were considered "converged" when the largest absolute matrix element of the matrix commutator FDS - SDF was smaller than $5 \times 10^{-4}$. This particular choice of convergence threshold is however not critical for the reported results, since the calculations that failed to converge due to vanishing gaps were typically very far from reaching this criterion. 


\section{A. Molecules from the protein data bank}

Table I shows computed HOMO-LUMO gaps for a set of protein-like molecular systems with geometries taken from the protein data bank (PDB) [9]. In cases where the PDB file contains more than one structure, the one labeled "model 1" was used. The 17 structures in Table I were selected in order to give examples of various types of protein-like systems, with the requirement that positions of hydrogen atoms should be included in the PDB file. The net charge of each molecule, shown in the fourth column in Table I, was chosen after performing a set of HF/3-21G calculations for different charges. For each system, the charge that gave the largest HOMO-LUMO gap was chosen. Calculations were performed using six different KS-DFT functionals as well as HF. The employed density functionals include the pure functionals LDA (SVWN5), BLYP, and PBE as well as the hybrid functionals B3LYP, PBE0, and BHandHLYP with HF exchange fractions of $20 \%, 25 \%$, and $50 \%$, respectively. The Gaussian basis set $6-31 \mathrm{G}^{* *}$ was used.

The most important conclusion from the results shown in Table I is that in many cases, calculations using pure functionals fail to converge for molecules larger than a few hundred atoms. Note that the blank spaces in the columns for BLYP, PBE, and LDA indicate not only that no gap value was obtained, but that those calculations did not give any meaningful results at all since they failed to converge.

For the calculations that did converge, the computed HOMO-LUMO gaps are strongly correlated to the fraction of HF exchange included in the functional, with a large fraction of HF exchange giving a large gap. Thus, for each molecule, the HF method yields the largest HOMOLUMO gap, while the BHandHLYP functional consistently gives a larger gap than PBE0 and B3LYP. The pure functionals give much smaller gaps; in many cases, no converged results were obtained for the pure functionals due to vanishing HOMO-LUMO gaps. For the 1RVS system, the B3LYP and PBE0 calculations also failed to converge.

To check the basis set dependence, calculations with larger basis sets were also performed for the smaller systems. The larger basis set results indicate that the computed HOMO-LUMO gaps are not critically dependent on the basis set. For example, calculations using the cc-pVTZ basis set for the 1BFZ, 1EVC, 1PLW, and 2P7R molecules gave HOMO-LUMO gaps that differed by less than $15 \%$ compared to the $6-31 G^{* *}$ results. In some cases, a larger basis set gives a smaller gap.

Calculations using the smaller basis set $3-21 \mathrm{G}$ were also performed. Those results indicate 


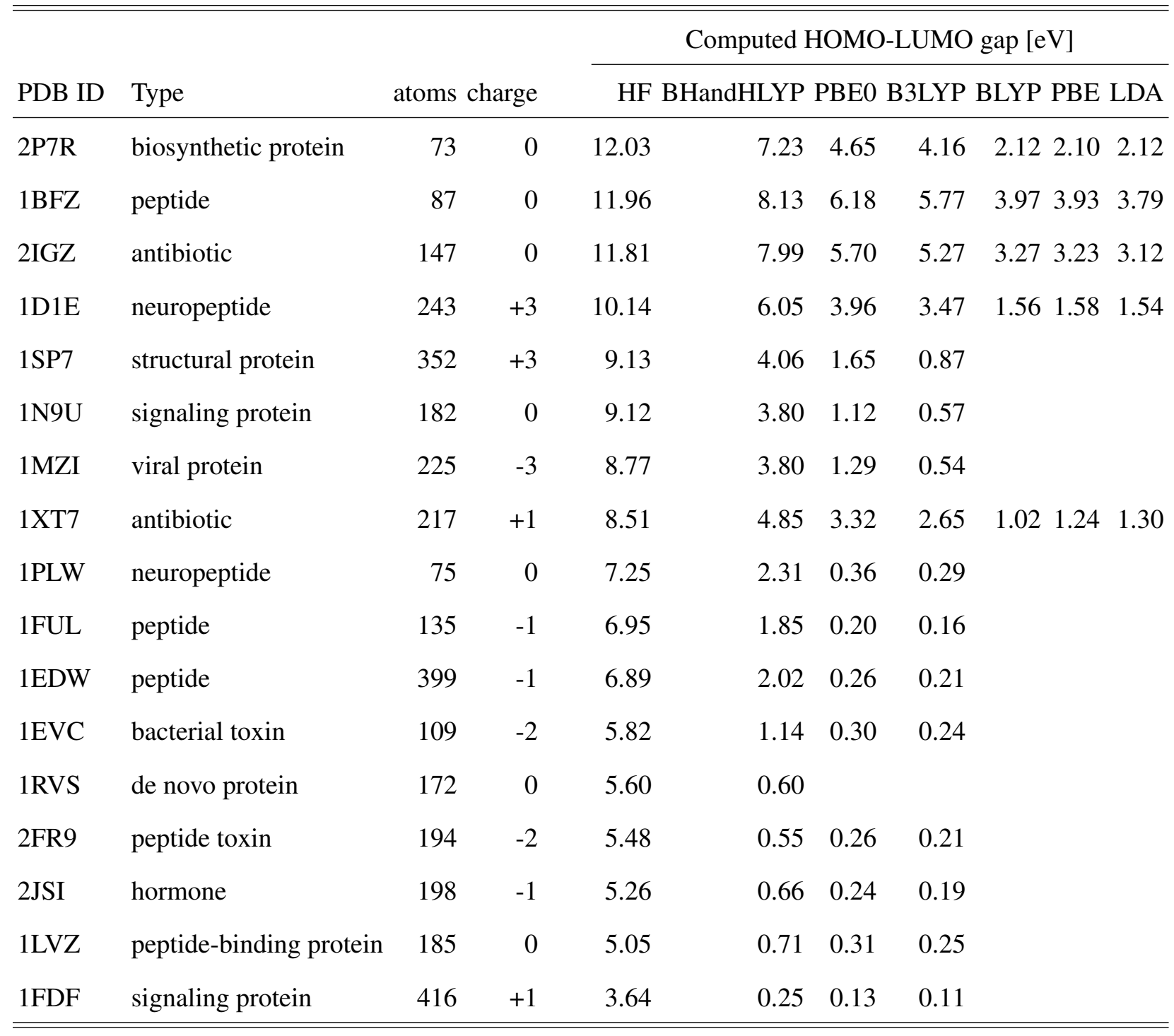

TABLE I: Results of HF and KS-DFT calculations using the Ergo program on a set of protein-like molecules. Basis set: 6-31G**. Blank space indicates that no converged result was obtained.

that already the 3-21G basis set gives similar gaps and convergence behavior for the different functionals.

\section{B. Size dependence}

The results in Table I indicate that the convergence problems due to small gaps are to a large extent system dependent. However, for even smaller protein-like fragments, consisting of only a few amino acids, calculations typically converge without problems also for pure functionals. Therefore there is reason to believe that that the convergence problems increase with increasing 


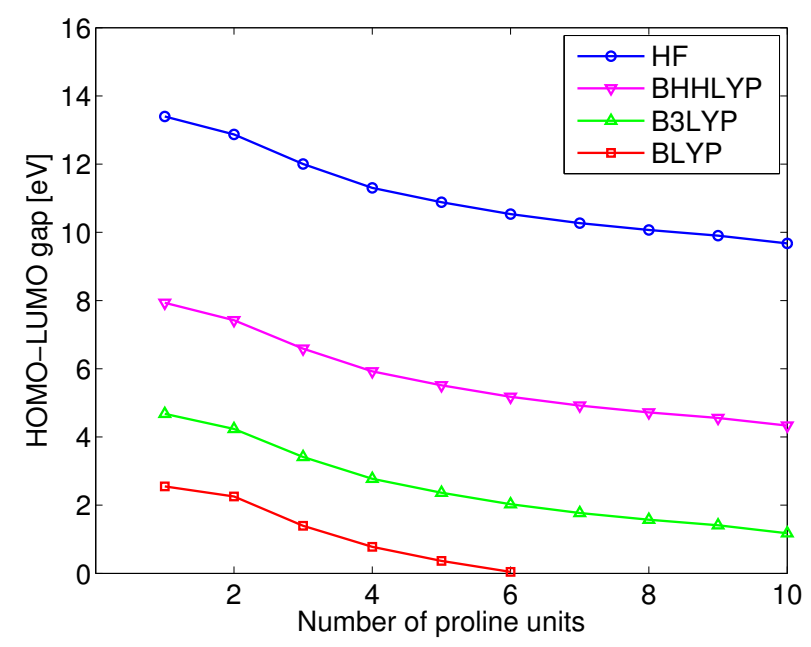

FIG. 1: Computed HOMO-LUMO gaps for polyproline I helix molecules. Basis set: $6-31 \mathrm{G}^{* *}$. The BLYP calculations for helices with 7-10 proline units failed to converge.

molecular size.

To further assess the size dependence, calculations were also performed for a sequence of polyproline I helix molecules of increasing length. The model helix geometries were generated using the Gabedit program [10], applying the "Build Polypeptide" function with the "Polyproline I" conformation followed by the "add hydrogens" command.

Computed HOMO-LUMO gaps for the polyproline I helix systems obtained using the KSDFT functionals BLYP, B3LYP, and BHandHLYP as well as HF are shown in Figure 1. The size dependence is clearly seen: for any given functional, the computed HOMO-LUMO gap decreases with increasing helix length, and because the computed gaps for pure functionals are so small, those calculations fail to converge for sizes larger than six proline units.

As seen in Figure 1, the problem of vanishing HOMO-LUMO gaps is in this case clearly related to the system size. However, the system size is not the only important factor. For example, performing the corresponding test calculations for helices in the polyproline II conformation gives sizable gaps even for very large systems. Apparently the problem of vanishing gaps is not seen for the fairly stretched out polyproline II helices, but the problem does appear for the more compact polyproline I conformation. 


\section{Including solvent water molecules}

The calculations in sections III A and III B were done for isolated protein-like systems without any surrounding water molecules. This is not completely realistic, since in real biological systems protein molecules are typically dissolved in water, and the solvent water molecules can have a significant effect on both the molecular geometry and the electronic structure of the protein. In this section, we consider the effect of explicitly including solvent water molecules when performing KS-DFT calculations for protein-like systems.

Since structures from the PDB in general do not include solvent molecules, model structures including solvent molecules were generated by molecular dynamics (MD) simulations at standard temperature and pressure using the Gromacs program [11]. The AMBER03 force field and the TIP3P water model were used. The MD simulations were done with the "position restraints" option in the Gromacs program, thus keeping the protein geometry reasonably close to the original geometry from the PDB, but allowing some motion and complete freedom of the surrounding solvent water molecules.

MD simulations were done for four of the systems from Section III A: 1FUL, 1LVZ, 1PLW, and 1RVS. For each of them, a number of MD runs were performed, generating ten uncorrelated MD snapshots. From each snapshot a model system with solvent was created by including all water molecules within $4 \AA$ from the solute. For comparison, corresponding model structures without solvent were also generated for the same set of MD snapshots. The structures without solvent differ slightly from the original PDB structures as the molecules moved during the MD simulations.

Figure 2 shows computed HOMO-LUMO gaps for the model systems generated from MD simulations. To reduce the computational effort, these calculations were done using the $3-21 \mathrm{G}$ basis set. Comparisons to larger basis set calculations done for a few cases indicate that the effect of this limited basis set is not critical; qualitatively similar results would probably be obtained with a larger basis set.

Figure 2(a) shows computed gaps for structures without surrounding solvent molecules. As can be expected from the results of Section III A, the BLYP calculations here give vanishing gaps and therefore fail to converge.

Figure 2(b) shows that the inclusion of explicit solvent molecules in the calculation in general gives a larger gap. However, in several cases the BLYP calculations still fail to converge. There 


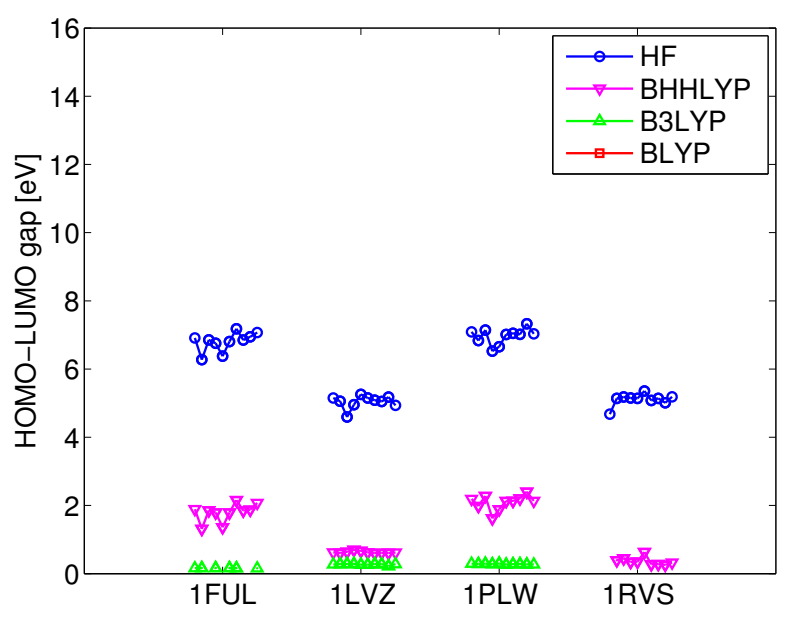

(a) Without surrounding water molecules

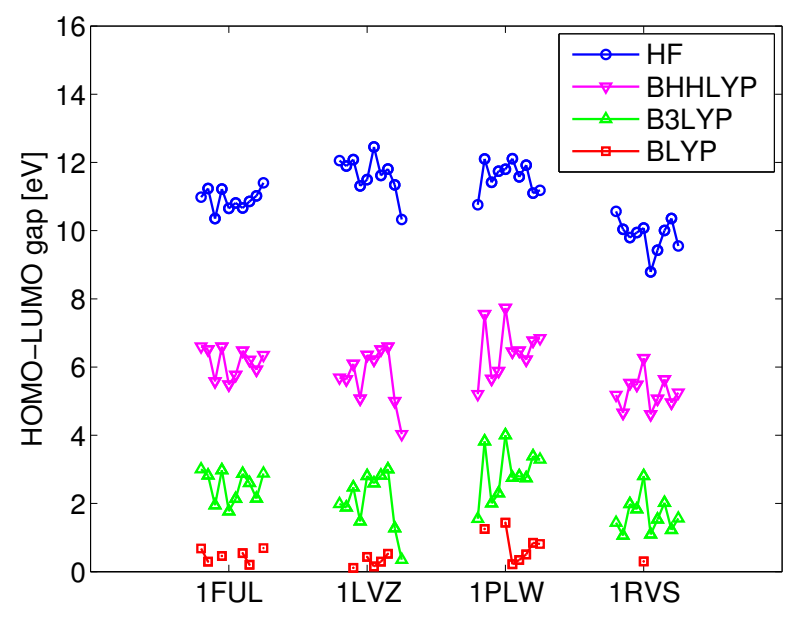

(b) With surrounding water molecules

FIG. 2: Computed HOMO-LUMO gaps for protein-like systems without and with surrounding water molecules. Basis set: 3-21G. Several of the BLYP calculations failed to converge even with surrounding water molecules.

is some randomness; BLYP calculations may or may not converge depending on the positions of included solvent molecules in that particular MD snapshot.

In the test calculations presented in Figure 2(b), water molecules up to $4 \AA$ from the solute were included. One may of course include more solvent molecules, but doing so does not seem to solve the problem. In fact, vanishing gaps for pure functionals is a problem also when considering water clusters, as shown in Figure 3. The water cluster geometries were generated by including all water molecules within a certain radius from a snapshot from an MD simulation at standard temperature and pressure. The problem of pure functionals giving vanishing gaps for water clusters was reported previously [12].

The computed gaps in Figure 3 decrease rather drastically at 13-16 $\AA$ radius, but this is a coincidence for the particular MD snapshot considered here; if continuing to larger clusters using $\mathrm{HF}$ or hybrid functionals the gaps tend to stabilize $[13,14]$. However, pure functionals are not straightforwardly applicable for water clusters generated in this way. Therefore, embedding a protein-like molecule in water by including explicit water molecules up to some radius cannot be expected to solve the convergence problems due to vanishing gaps. In order to achieve converged results with pure functionals, some modeling needs to be done of the other water molecules, outside the domain of the electronic structure calculation, as will be seen in the next section. 


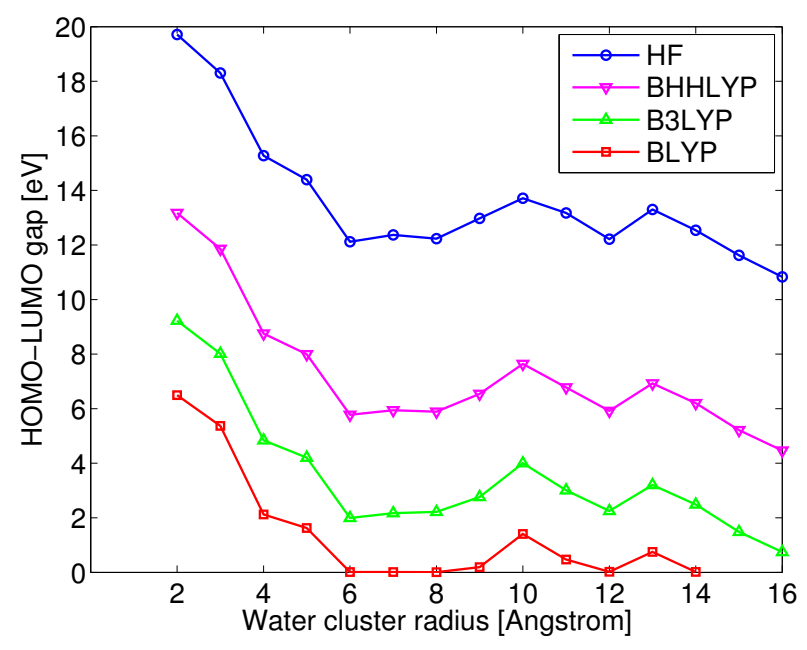

FIG. 3: Computed HOMO-LUMO gaps for water clusters. Basis set: 3-21G. The BLYP calculations for water clusters of radius 15-16 Å failed to converge.

\section{Including point charges representing solvent water molecules outside computational domain}

Previous work by Cabral do Couto et al. [15] has shown that for water clusters extracted from a larger simulation, orbital energies are strongly affected by the water molecules surrounding the clusters, and that such surface effects can to some extent be corrected for by including point charges representing the surrounding molecules. Cabral do Couto et al. found that HOMOLUMO gaps are significantly increased when adding point charges representing surrounding water molecules. In this section, the approach of adding such point charges is applied to the case of protein molecules embedded in water.

The test systems used in this section are the same as those in Section III C except that now water molecules outside the electronic structure calculation domain are included via point charges. These "outer" water molecules are not explicitly included in the electronic structure calculation, but they are represented by point charges corresponding to their simple point charge (SPC) distribution. That is, oxygen and hydrogen atoms are represented by point charges of -0.82 and +0.41 , respectively. Outer water molecules up to $10 \AA$ away from the studied system were included. This gives a large number of point charges (for 1RVS, around 4800 point charges were used) but the extra computational effort is anyway small since the point charges only affect the core Hamiltonian matrix. The expensive Coulomb, HF exchange, and exchange-correlation parts of the calculation are not affected by the added point charges. 


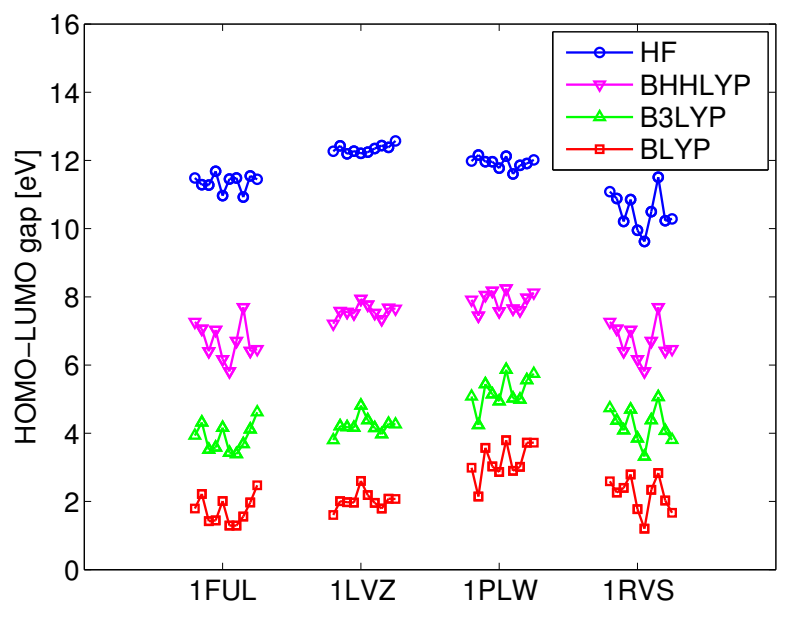

(a) Without surrounding water molecules

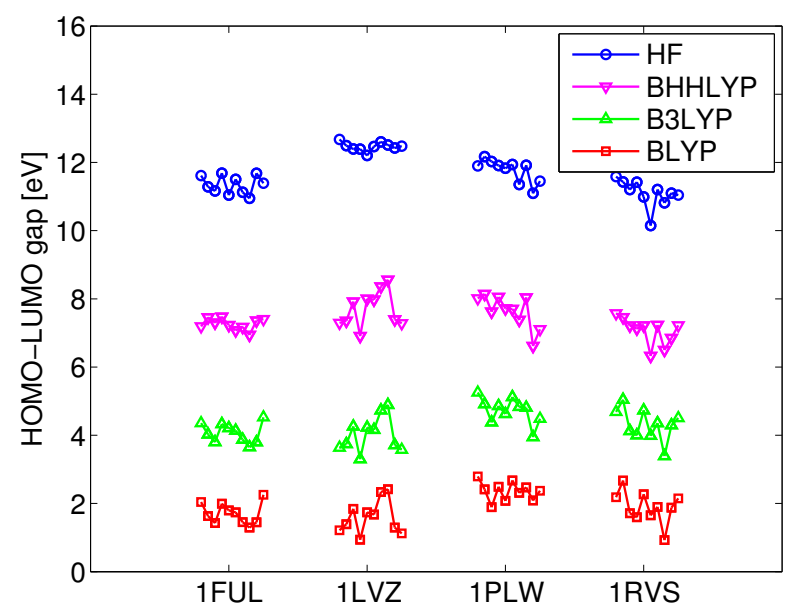

(b) With surrounding water molecules

FIG. 4: Computed HOMO-LUMO gaps for protein-like systems without and with surrounding water molecules. Basis set: 3-21G. In both cases, water molecules outside the computational domain were represented by SPC point charges.

Figure 4 shows computed HOMO-LUMO gaps for the same systems as in Figure 2, but now including SPC point charges as described above. Note that in the calculations shown in Figure 4(b), water molecules are included in two ways: water molecules up to $4 \AA ̊$ from the solute are explicitly included in the electronic structure calculation, and additional water molecules between 4 and $14 \AA$ away from the solute are represented by point charges.

Judging from Figure 4, the approach of including point charges representing water molecules outside the electronic structure calculation domain appears to solve the convergence problems for pure functionals: when point charges are included in this way, BLYP calculations give HOMOLUMO gaps of more than $0.9 \mathrm{eV}$ in all studied cases. This approach also gives convergence for the polyproline I helix systems considered in Section III B.

Thus, it appears that despite the discouraging results of sections III A and III B, calculations using pure functionals can be done for protein-like systems provided that surrounding solvent water molecules are accounted for by somehow taking their charge distribution into account. If solvent water molecules are explicitly included in the electronic structure calculation, surface effects must anyway be handled by including the charge distribution water molecules further away.

In this section, surface effects were handled using point charges in the same way as in the work of Cabral do Couto et al [15]. This is easily done from an implementation point of view 
and required only a minor modification to the Ergo program [8] that was used to perform the calculations. Another way of taking effects of the surrounding water into account would be to use a polarizable continuum model, although that possibility was not explored here.

The point charge embedding approach was here considered as a tool to obtain a converged solution. Of course, such point charges are a very crude approximation of the solvent atoms they are supposed to represent. One should therefore be careful when interpreting results of such calculations, in particular regarding the electronic structure near the boundary where point charges were added.

\section{CONCLUDING REMARKS}

All calculations reported in this work were performed using Gaussian basis sets, far from the basis set limit. To better assess the basis set dependence, it would be desirable to also perform calculations with other types of basis sets, e.g. plane waves.

The results obtained here for protein fragments are in line with previous findings that pure KSDFT functionals give vanishing gaps for large polypeptide and water cluster systems [14]. Also, vanishing PBE gaps have been reported for plane-wave calculations on semiconductors [16].

Although the problem of pure KS-DFT functionals underestimating HOMO-LUMO gaps is well known in the literature [17-22], to the author's best knowledge the resulting convergence problems in self-consistency based calculations for protein-like molecules has received little, if any, attention previously.

It should be noted that the calculations reported in this work were done for finite model systems. That is, periodic boundary conditions were not used. When using a finite model system to describe a protein in water solution, the domain must be truncated somewhere, and it is then important to handle surface effects in some way, for example as described in Section III D. In a periodic calculation there is no boundary and thus no surface effects to worry about. Periodic calculations using pure KS-DFT for proteins have been reported for example by Sulpizi et al. [23].

The calculations in this work were all performed using the self-consistency approach, as described in Section II. Therefore, a non-vanishing HOMO-LUMO gap was here necessary to achieve convergence. It should be noted that other optimization schemes for KS-DFT calculations exist, where a parametrization is used that ensures that the density matrix stays idempotent (and has the correct number of electrons), but where there is no guarantee that the orbitals defin- 
ing the density are the ones having the lowest orbital energies. Then, a converged solution could in principle be found even if the gap vanishes. However, such approaches were not used in the present work.

In the calculations reported in this work, significant effort was made to reduce the risk that the reported results are dependent on any particular choice of starting guess density. For cases that turned out to be difficult to converge, repeated calculations using several different starting guesses were tried, including densities obtained with other functionals and other basis sets. In those cases where "convergence failure" is reported, this does not mean only that one particular calculation failed to converge, but that all calculation attempts using various starting guesses failed.

All results reported in Section III are from spin-restricted (closed shell) calculations. Additional spin-unrestricted calculations with different alpha- and beta-spin densities as starting guesses were performed for many of the studied cases. In those cases, spin-unrestricted calculations did not resolve the convergence problems.

Test calculations using level shifting [24] were performed for a few of the difficult cases in sections III A and III B. If employing a large enough shift, a converged result can sometimes be obtained. However, if the resulting density is used as a starting guess for a calculation without any level shift, different orbitals are occupied and convergence is not obtained. Also, the calculations with level shifting are very sensitive to the starting guess. In cases where the usual self-consistency based approach (without level shifting) fails due to vanishing gaps, calculations employing level shifting may converge to any of many possible final results with small differences in energy, depending on the starting guess. Such solutions found using level shifting do typically not obey the aufbau principle; that is, the occupied orbitals are not the ones having the lowest orbital energies. This suggests that proper aufbau solutions to the standard Kohn-Sham model may not exist for these cases; compare for example to the case of chromium carbide considered by Kudin et al [25]. In any case, using level shifting does not seem to be a satisfactory solution to the convergence problems, since the final result then becomes heavily dependent on the starting guess.

Another way to achieve convergence in difficult cases would be to employ fractional finitetemperature occupation numbers in the same way as in calculations for metals [26, 27]. Alternatively, instead of standard KS-DFT methods one may consider employing the extended KohnSham model [28], or using GW theory [29]. However, application of such methods goes beyond the scope of the present work.

Application of self-consistency based pure KS-DFT methods to protein-like molecules without 
including solvent often leads to convergence problems due to vanishing HOMO-LUMO gaps. Although such problems can be alleviated by including solvent molecules, they indicate that the applicability of such pure KS-DFT methods may be limited: if a protein-like system surrounded by air or vacuum is to be studied, it is unclear to what extent self-consistency based pure KS-DFT methods can be applied. Further investigation of this issue remains a subject of future work.

\section{Acknowledgements}

The author wishes to thank E. R. Davidson, E. H. Rubensson, and P. Sałek for helpful discussions. The computations were performed on resources provided by the Swedish National Infrastructure for Computing (SNIC) at High Performance Computing Center North (HPC2N) and Uppsala Multidisciplinary Center for Advanced Computational Science (UPPMAX).

[1] W. Kohn and L. J. Sham, Phys. Rev. 140, A1133 (1965).

[2] S. Goedecker, Rev. Mod. Phys. 71, 1085 (1999).

[3] P. Mori-Sánchez, Q. Wu, and W. Yang, J. Chem. Phys. 119, 11001 (2003).

[4] A. Szabo and N. S. Ostlund, Modern Quantum Chemistry: Introduction to Advanced Electronic Structure Theory (Dover Publications, Inc., Mineola, New York, 1996).

[5] M. C. Zerner and M. Hehenberger, Chem. Phys. Lett. 62, 550 (1979).

[6] P. Pulay, J. Comput. Chem. 3, 556 (1982).

[7] K. N. Kudin and G. E. Scuseria, Math. Model. Num. Anal. 41, 281 (2007).

[8] E. Rudberg, E. H. Rubensson, and P. Sałek, Ergo (version 3.51); a quantum chemistry program for large scale self-consistent field calculations (2011), URL http: / / www • ergoscf . org.

[9] The protein data bank, http: / / www.pdb.org.

[10] A. Allouche, J. Comput. Chem. 32, 174 (2011).

[11] B. Hess, C. Kutzner, D. van der Spoel, and E. Lindahl, J. Chem. Theory Comput. 4, 435 (2008).

[12] E. H. Rubensson and E. Rudberg, J. Comput. Chem. 32, 1411 (2011).

[13] E. Rudberg, E. H. Rubensson, and P. Sałek, J. Chem. Phys. 128, 184106 (2008).

[14] E. Rudberg, E. H. Rubensson, and P. Sałek, J. Chem. Theory Comput. 7, 340 (2011).

[15] P. Cabral do Couto, R. C. Guedes, and B. J. Costa Cabral, Braz. J. Phys. 34, 42 (2004). 
[16] P. Deák, B. Aradi, T. Frauenheim, E. Janzén, and A. Gali, Phys. Rev. B 81, 153203 (2010).

[17] J. P. Perdew and M. Levy, Phys. Rev. Lett. 51, 1884 (1983).

[18] R. W. Godby, M. Schlüter, and L. J. Sham, Phys. Rev. B 37, 10159 (1988).

[19] A. Seidl, A. Görling, P. Vogl, J. A. Majewski, and M. Levy, Phys. Rev. B 53, 3764 (1996).

[20] U. Salzner, J. B. Lagowski, P. G. Pickup, and R. A. Poirier, J. Comput. Chem. 18, 1943 (1997).

[21] S. Lany and A. Zunger, Phys. Rev. B 78, 235104 (2008).

[22] T. Stein, H. Eisenberg, L. Kronik, and R. Baer, Phys. Rev. Lett. 105, 266802 (2010).

[23] M. Sulpizi, S. Raugei, J. VandeVondele, P. Carloni, and M. Sprik, J. Phys. Chem. B 111, 3969 (2007).

[24] V. R. Saunders and I. H. Hillier, Int. J. Quantum Chem. 7, 699 (1973).

[25] K. N. Kudin, G. E. Scuseria, and E. Cancès, J. Chem. Phys. 116, 8255 (2002).

[26] N. Marzari, D. Vanderbilt, and M. C. Payne, Phys. Rev. Lett. 79, 1337 (1997).

[27] C. Freysoldt, S. Boeck, and J. Neugebauer, Phys. Rev. B 79, 241103 (2009).

[28] E. Cancès, J. Chem. Phys. 114, 10616 (2001).

[29] M. van Schilfgaarde, T. Kotani, and S. Faleev, Phys. Rev. Lett. 96, 226402 (2006). 